\title{
A NOTE ON VALUE DISTRIBUTION OF DIFFERENCE POLYNOMIALS
}

\author{
K. LIU ${ }^{凶}$ and I. LAINE
}

(Received 29 January 2009)

\begin{abstract}
In this paper, we investigate the value distribution of difference polynomials and prove some difference analogues of results of Hayman and the Brück conjecture.
\end{abstract}

2000 Mathematics subject classification: primary 30D35; secondary 39B32.

Keywords and phrases: shift, difference operator, finite order.

\section{Introduction}

In [5, Theorem 8] Hayman proved, among other results, that a differential polynomial $f^{n}+a f^{\prime}-b$ with constant coefficients $a, b$ admits infinitely many zeros, provided that $f$ is transcendental entire and $n \geq 3$ (or $n \geq 2$ if $b=0$ ). The main purpose of this paper is to establish a partial difference counterpart of the Hayman result by proving that whenever $f$ is a nonperiodic entire function of finite order, $c$ is a nonzero complex constant, and $f(z)^{n}+f(z+c)-f(z)-b$ has finitely many zeros, then $f$ must be a polynomial, provided $n \geq 3$ (or $n \geq 2$ if $b=0$ ). Simple examples show that the claim fails if $n=2$ (or if $n=1$ and $b=0$ ). A little more generally, we prove the following two theorems.

THEOREM 1.1. Let $f$ be a transcendental entire function of finite order $\rho$, not of period $c$, where $c$ is a nonzero complex constant. Then the difference polynomial $f(z)^{n}+f(z+c)-f(z)$ has infinitely many zeros in the complex plane, provided that $n \geq 2$.

THEOREM 1.2. Let $f$ be a transcendental entire function of finite order $\rho$, not of period $c$, where $c$ is a nonzero complex constant, and let $s(z)$ be a nonzero function, small compared to $f$. Then the difference polynomial $f(z)^{n}+f(z+c)-f(z)-$ $s(z)$ has infinitely many zeros in the complex plane, provided that $n \geq 3$.

The second author has been partially supported by the Academy of Finland grant 124954 .

(C) 2010 Australian Mathematical Publishing Association Inc. 0004-9727/2010 \$16.00 
Assuming that the shift constant $c$ is fixed, in what follows, we introduce the difference operator

$$
\Delta f=\Delta_{1} f:=f(z+c)-f(z)
$$

inductively defining

$$
\Delta_{n} f:=\Delta_{n-1}(\Delta f)
$$

for all natural numbers $n \geq 2$. We also prove the following theorem.

THEOREM 1.3. Let $f$ be a transcendental entire function of order $\rho(f)<2$ not having period $c$. If $f$ and $\Delta_{n} f$ share the value $0 C M$, then $\Delta_{n} f / f$ reduces to a nonzero constant.

REMARK 1.4. From the function $f(z)=A e^{z \log (c+1)}-((1-c) / c)$, where $c \in \mathbb{R} /\{0\}$, $c>-1$, and $A$ is an arbitrary constant, we get $(f(z+1)-f(z)-1) /(f(z)-1)=c$. The example suggests that $\left(\Delta_{n} f-a\right) /(f-a)$ may reduce to a nonzero constant, at least if $\rho(f)=1$ and $N(r, 1 / f) \neq S(r, f)$. We believe that $\left(\Delta_{n} f-a\right) /(f-a)$ reduces to a nonzero constant, provided that $f$ is a transcendental entire function of order $1<\rho<\infty$ not having period $c$, and that $f$ and $\Delta_{n} f$ share $\mathrm{CM}$ a nonzero value $a \in \mathbb{C}$. Unfortunately, we have not succeeded in proving this.

Observe that Theorem 1.3 above and the preceding remark are closely related to what could be called a difference counterpart of the Brück conjecture [1]. For a related result in the difference setting, see [7, Theorem 2.1].

In what follows, we assume that the reader is familiar with the basic notation and results in the Nevanlinna value distribution theory $[6,8]$ as well as in the uniqueness theory of entire and meromorphic functions [9].

\section{Three preliminary lemmas}

In the proof of Theorem 1.1 below, we make use of three key lemmas. For the convenience of the reader, we recall these lemmas here.

The following result is due to Yang and Yi (see [9, Theorem 1.56]).

LEMMA 2.1. Let $f_{1}, f_{2}, f_{3}$ be meromorphic functions such that $f_{1}$ is not constant. If $f_{1}+f_{2}+f_{3}=1$ and if

$$
\sum_{j=1}^{3} N\left(r, 1 / f_{j}\right)+2 \sum_{j=1}^{3} \bar{N}\left(r, f_{j}\right)<(\lambda+o(1)) T(r),
$$

where $\lambda<1$ and $T(r):=\max _{1 \leq j \leq 3} T\left(r, f_{j}\right)$, then either $f_{2}=1$ or $f_{3}=1$.

Next, we need to apply the well-known Clunie lemma (see [3]). The following version [8, Theorem 2.4.2], slightly more general than the original one, has been reinvented several times in the literature. 
LEMMA 2.2. Let $f$ be a transcendental meromorphic solution of

$$
f^{n} P(z, f)=Q(z, f),
$$

where $P(z, f), Q(z, f)$ are differential polynomials in $f$ and its derivatives with small meromorphic coefficients $a_{\lambda}, \lambda \in I$, in the sense of $m\left(r, a_{\lambda}\right)=S(r, f)$ for all $\lambda \in I$. If the total degree of $Q(z, f)$ as a polynomial in $f$ and its derivatives is $n$ or less, then

$$
m(r, P(z, f))=S(r, f) .
$$

Finally, we recall a variant on [2, Corollary 2.5] of the difference counterpart of the logarithmic derivative lemma (see also [4, Corollary 2.2]).

LEMMA 2.3. Let $f$ be a meromorphic function of finite order $\rho$, and let $\eta_{1}, \eta_{2}$ be two distinct complex numbers. Then for any $\varepsilon>0$, we have

$$
m\left(r, \frac{f\left(z+\eta_{1}\right)}{f\left(z+\eta_{2}\right)}\right)=O\left(r^{\rho-1+\varepsilon}\right) .
$$

\section{Proof of Theorem 1.1}

Suppose that $f(z)^{n}+f(z+c)-f(z)$ admits finitely many zeros only. Then there are two polynomials $r(z), p(z)$ such that

$$
f(z)^{n}+f(z+c)-f(z)=r(z) e^{p(z)} .
$$

Differentiating (3.1) and eliminating $e^{p(z)}$, we obtain

$$
\begin{aligned}
& f(z)^{n-1}\left(n f^{\prime}(z)-\left(p^{\prime}(z)+\frac{r^{\prime}(z)}{r(z)}\right) f(z)\right) \\
& =f^{\prime}(z)-f^{\prime}(z+c)+\left(p^{\prime}(z)+\frac{r^{\prime}(z)}{r(z)}\right)(f(z+c)-f(z)) .
\end{aligned}
$$

If $n f^{\prime}-\left(p^{\prime}+r^{\prime} / r\right) f$ vanishes identically, then $f=s e^{q}$, where $s, q$ are polynomials. Substituting this into (3.1), we get

$$
\begin{aligned}
f_{1}+f_{2}+f_{3} & =s(z)^{n-1} e^{(n-1) q(z)}+\frac{s(z+c)}{s(z)} e^{q(z+c)-q(z)}-\frac{r(z)}{s(z)} e^{p(z)-q(z)} \\
& \equiv 1
\end{aligned}
$$

Since $f$ is nonconstant, we have either $f_{2}=1$ or $f_{3}=1$ by Lemma 2.1. If $f_{2}=1$, then $f$ has to be periodic, which contradicts our assumptions. If $f_{3}=1$, we see that $f=-r e^{p}$. Therefore, (3.1) takes the form $f(z)^{n}+f(z+c)=0$. Now substituting $f=s e^{q}$ in this identity, we get

$$
s(z)^{n-1} e^{(n-1) q(z)}+\frac{s(z+c)}{s(z)} \frac{e^{q(z+c)}}{e^{q(z)}}=0 .
$$


Applying the lemma of logarithmic differences (Lemma 2.3), we conclude that

$$
T\left(r, e^{q}\right)=m\left(r, e^{q}\right)=S\left(r, e^{q}\right),
$$

a contradiction.

It remains to consider the case where $n f^{\prime}-\left(p^{\prime}+r^{\prime} / r\right) f \neq 0$. First, if $n \geq 3$, we may rewrite (3.2) in the form

$$
\begin{aligned}
f(z)^{n-2}\left(n f^{\prime}(z)-\left(p^{\prime}(z)+\frac{r^{\prime}(z)}{r(z)}\right) f(z)\right) \\
\quad=\frac{f^{\prime}(z)}{f(z)}-\frac{f^{\prime}(z+c)}{f(z+c)} \frac{f(z+c)}{f(z)}+\left(p^{\prime}(z)+\frac{r^{\prime}(z)}{r(z)}\right)\left(\frac{f(z+c)}{f(z)}-1\right) .
\end{aligned}
$$

By the Clunie lemma (Lemma 2.2), we conclude that

$$
T\left(r, n f^{\prime}-\left(p^{\prime}+\frac{r^{\prime}}{r}\right) f\right)=O\left(r^{\rho-1+\varepsilon}\right)+S(r, f)
$$

and

$$
T\left(r, f\left(n f^{\prime}-\left(p^{\prime}+\frac{r^{\prime}}{r}\right) f\right)\right)=O\left(r^{\rho-1+\varepsilon}\right)+S(r, f)
$$

as well. Combining these two estimates, we obtain

$$
T(r, f)=O\left(r^{\rho-1+\varepsilon}\right)+S(r, f),
$$

a contradiction.

Therefore, we may finally assume that $n=2$ and $2 f^{\prime}-\left(p^{\prime}+r^{\prime} / r\right) f \neq 0$. Clearly, there exist polynomials $r(z), p(z)$ such that

$$
f(z)^{2}+f(z+c)-f(z)=r(z) e^{p(z)} .
$$

Differentiating (3.4) and eliminating $e^{p(z)}$ now results in

$$
\begin{aligned}
& f(z)\left(2 f^{\prime}(z)-\left(p^{\prime}(z)+\frac{r^{\prime}(z)}{r(z)}\right) f(z)\right) \\
& \quad=f^{\prime}(z+c)-f^{\prime}(z)-\left(p^{\prime}(z)+\frac{r^{\prime}(z)}{r(z)}\right)(f(z+c)-f(z)) .
\end{aligned}
$$

Dividing (3.5) by $f(z)$ and applying the standard lemma of the logarithmic derivative as well as its difference counterpart (see [4]), we conclude that $\psi(z)$ is small compared to $f(z)$ in the usual sense of Nevanlinna theory. By differentiation of $\psi(z)$ we obtain

$$
2 f^{\prime \prime}-\left(p^{\prime}+\frac{r^{\prime}}{r}\right) f^{\prime}-\left(p^{\prime}+\frac{r^{\prime}}{r}\right)^{\prime} f=\psi^{\prime}=\frac{\psi^{\prime}}{\psi} \psi=\frac{\psi^{\prime}}{\psi}\left(2 f^{\prime}-\left(p^{\prime}+\frac{r^{\prime}}{r}\right) f\right),
$$


and so

$$
2 f^{\prime \prime}-\left(p^{\prime}+\frac{r^{\prime}}{r}+2 \frac{\psi^{\prime}}{\psi}\right) f^{\prime}-\left(p^{\prime \prime}-p^{\prime} \frac{\psi^{\prime}}{\psi}+\left(\frac{r^{\prime}}{r}\right)^{\prime}-\frac{r^{\prime}}{r} \frac{\psi^{\prime}}{\psi}\right) f=0
$$

This may be written as

$$
\begin{aligned}
2\left(\frac{f^{\prime}}{f}\right)^{\prime}+2\left(\frac{f^{\prime}}{f}\right)^{2}-\left(p^{\prime}+\frac{r^{\prime}}{r}+2 \frac{\psi^{\prime}}{\psi}\right) \frac{f^{\prime}}{f} \\
-\left(p^{\prime \prime}-p^{\prime} \frac{\psi^{\prime}}{\psi}+\left(\frac{r^{\prime}}{r}\right)^{\prime}-\frac{r^{\prime}}{r} \frac{\psi^{\prime}}{\psi}\right)=0 .
\end{aligned}
$$

We next proceed to show that $f$ has a few zeros only in the sense that $N(r, 1 / f)=$ $S(r, f)$. Suppose that $f\left(z_{0}\right)=0$. Since $r$ is a polynomial, we may assume that $r\left(z_{0}\right) \neq 0$. Suppose first that $\psi\left(z_{0}\right) \neq 0$. Then the double pole terms on the left-hand side of (3.6) cancel only if the zero of $f$ is a simple one. To avoid a contradiction on the left-hand side of (3.6), we must have that $p^{\prime}+\left(r^{\prime} / r\right)+2\left(\psi^{\prime} / \psi\right)$ vanishes at $z_{0}$. Therefore, the points where $f\left(z_{0}\right)=0, r\left(z_{0}\right) \neq 0$ and $\psi\left(z_{0}\right) \neq 0$ make a contribution of $S(r, f)$ to $N(r, 1 / f)$. Assume, finally, that $f\left(z_{0}\right)=0, r\left(z_{0}\right) \neq 0$ and $\psi\left(z_{0}\right)=0$. Let $\alpha$ and $\beta$, respectively, be the multiplicities of the zeros of $f$ and $\psi$ at $z_{0}$. Now substitution into (3.6) results in $\alpha=1+\beta \leq 2 \beta$, again implying a contribution of $S(r, f)$ to $N(r, 1 / f)$. Therefore, $f(z)$ takes the form $f(z)=\phi(z) e^{q(z)}$, where $q(z)$ is a polynomial and $N(r, 1 / \phi)=S(r, f)$. Plugging this expression into (3.4), we obtain

$$
\phi(z)^{2} e^{2 q(z)}+\phi(z+c) e^{q(z+c)}-\phi(z) e^{q(z)}=r(z) e^{p(z)},
$$

which may be written in the form

$$
\phi(z) e^{q(z)}+\frac{\phi(z+c)}{\phi(z)} e^{q(z+c)-q(z)}-\frac{r(z)}{\phi(z)} e^{p(z)-q(z)}=1 .
$$

Recalling Lemma 2.1, either $f_{2}(z):=(\phi(z+c) / \phi(z)) e^{q(z+c)-q(z)} \equiv 1$ or $f_{3}(z):=$ $-(r(z) / \phi(z)) e^{p(z)-q(z)} \equiv 1$. If $f_{2}(z) \equiv 1$, then $f$ is periodic, a contradiction. Finally, if $f_{3}(z) \equiv 1$, then $f(z) \equiv-r(z) e^{p(z)}$. Substituting into (3.4), we get

$$
\left(\phi(z) e^{p(z)}\right)^{2}-\phi(z+c) e^{p(z+c)}=0 .
$$

Writing (3.8) in the form

$$
\phi(z) e^{p(z)}-\frac{\phi(z+c)}{\phi(z)} \frac{e^{p(z+c)}}{e^{p(z)}}=0,
$$

and recalling again the lemma of logarithmic differences from [4], we conclude that

$$
T\left(r, e^{p}\right)=m\left(r, e^{p}\right)=S\left(r, e^{p}\right),
$$

a contradiction, completing the proof.

REMARK 3.1. The basic exponential function $e^{z}$ may be used to show that the claim of Theorem 1.1 fails if $n=1$. In fact, taking $c \neq 2 \pi i m$ for an arbitrary natural number $m$, we have that $f(z)+f(z+c)-f(z)=e^{c} e^{z}$ has no zeros. 


\section{Proof of Theorem 1.2}

Suppose that $f(z)^{n}+f(z+c)-f(z)-s(z)$ has finitely many zeros. Then there exist two polynomials $r(z)$ and $p(z)$ such that

$$
f(z)^{n}+f(z+c)-f(z)-s(z)=r(z) e^{p(z)} .
$$

Differentiating (4.1) and eliminating $e^{p(z)}$, we obtain

$$
\begin{aligned}
& f(z)^{n-1}\left(n f^{\prime}(z)-\left(p^{\prime}(z)+\frac{r^{\prime}(z)}{r(z)}\right) f(z)\right)=f^{\prime}(z)-f^{\prime}(z+c)+s^{\prime}(z) \\
& +\left(p^{\prime}(z)+\frac{r^{\prime}(z)}{r(z)}\right)(f(z+c)-f(z)-s(z)) .
\end{aligned}
$$

If $n f^{\prime}-\left(p^{\prime}+r^{\prime} / r\right) f$ vanishes identically, then $f^{n}=C r e^{p}$. Writing $f=h e^{p / n}$, where $h$ is a polynomial, and substituting $f$ into (4.1), we get

$$
(C-1) r(z) e^{p(z)}+h(z+c) e^{p(z+c) / n}-h(z) e^{p(z) / n}-s(z) \equiv 0 .
$$

Let $g=e^{p / n}$. If $C \neq 1$, then we obtain, from (4.3) and Lemma 2.3, that

$$
n T(r, g) \leq 2 T(r, g)+S(r, g),
$$

in contradiction to $n \geq 3$.

Therefore, we must have $C=1$. From (4.3), we get

$$
h(z+c) e^{p(z+c) / n}-h(z) e^{p(z) / n}-s(z) \equiv 0 .
$$

Applying the second main theorem for three small targets [6, Theorem 2.5] we get

$$
\begin{aligned}
T(r, f) & \leq \bar{N}(r, f)+\bar{N}\left(r, \frac{1}{f+s(z)}\right)+\bar{N}\left(r, \frac{1}{f}\right)+S(r, f) \\
& =\bar{N}\left(r, \frac{1}{f(z+c)}\right)+S(r, f) \\
& =S(r, f),
\end{aligned}
$$

a contradiction.

It remains to treat the case where $n f^{\prime}-\left(p^{\prime}+r^{\prime} / r\right) f$ is not vanishing identically. Since $n \geq 3$, we may apply the Lemma 2.2 and (4.2) to conclude that

$$
T\left(r, n f^{\prime}-\left(p^{\prime}+\frac{r^{\prime}}{r}\right) f\right)=O\left(r^{\rho-1+\varepsilon}\right)+S(r, f)
$$

and

$$
T\left(r, f\left(n f^{\prime}-\left(p^{\prime}+\frac{r^{\prime}}{r}\right) f\right)\right)=O\left(r^{\rho-1+\varepsilon}\right)+S(r, f)
$$

as well. Combining these two estimates, we obtain

$$
T(r, f)=O\left(r^{\rho-1+\varepsilon}\right)+S(r, f),
$$

a contradiction, completing the proof. 
REMARK 4.1. Theorem 1.2 is not true if $n=2$. This can be seen by considering $f(z)=e^{z}+1, c=\pi i$. Then $f(z)^{2}+f(z+c)-f(z)-1=e^{2 z}$ has no zeros.

\section{Proof of Theorem 1.3}

By assumptions, there exists a polynomial $Q(z)$ such that

$$
\frac{\Delta_{n} f}{f}=e^{Q} \text {. }
$$

We can immediately verify that

$$
\Delta_{n} f(z)=f(z+n c)+A_{n-1} f(z+(n-1) c)+\cdots+A_{0} f(z),
$$

where $A_{0}, \ldots, A_{n-1}$ are constants. We may now write (5.1) in the form

$$
e^{Q(z)}-A_{0}=\frac{f(z+n c)}{f(z)}+A_{n-1} \frac{f(z+(n-1) c)}{f(z)}+\cdots+A_{1} \frac{f(z+c)}{f(z)} .
$$

Given $\varepsilon>0$ small enough to satisfy $\rho+\varepsilon<2$, we obtain, by Lemma 2.3, that

$$
T\left(r, e^{Q}\right)=m\left(r, e^{Q}\right)=O\left(r^{\rho-1+\varepsilon}\right)+O(1) .
$$

Therefore, the polynomial $Q$ reduces to a constant, completing the proof.

\section{Acknowledgement}

The second author wishes to thank Rod Halburd for his hospitality during a short visit to UCL London, where part of his contribution to this paper was completed.

\section{References}

[1] R. Brück, 'On entire functions which share one value CM with their first derivative', Results in Math. 30 (1996), 21-24.

[2] Y. M. Chiang and S. J. Feng, 'On the Nevanlinna characteristic of $f(z+\eta)$ and difference equations in the complex plane', The Ramanujan J. 16 (2008), 105-129.

[3] J. Clunie, 'On integral and meromorphic functions', J. Lond. Math. Soc. 37 (1962), 17-27.

[4] R. G. Halburd and R. J. Korhonen, 'Difference analogue of the lemma on the logarithmic derivative with applications to difference equations', J. Math. Anal. Appl. 314 (2006), 477-487.

[5] W. K. Hayman, 'Picard values of meromorphic functions and their derivatives', Ann. of Math. (2) 70 (1959), 9-42.

[6] W. K. Hayman, Meromorphic Functions (Clarendon Press, Oxford, 1964).

[7] J. Heittokangas, R. Korhonen, I. Laine, J. Rieppo and J. L. Zhang, 'Value sharing results for shifts of meromorphic functions, and sufficient conditions for periodicity', J. Math. Anal. Appl. 355 (2009), 352-363.

[8] I. Laine, Nevanlinna Theory and Complex Differential Equations (Walter de Gruyter, Berlin, 1993).

[9] C.-C. Yang and H.-X. Yi, Uniqueness Theory of Meromorphic Functions (Kluwer, Dordrecht, 2003). 
K. LIU, Department of Mathematics, Nanchang University, Nanchang, Jiangxi, 330031, PR China

and

Current address: Department of Mathematics, University of Joensuu, PO Box 111, FI-80101 Joensuu, Finland

e-mail: liukai418@126.com

I. LAINE, Department of Mathematics, University of Joensuu, PO Box 111, FI-80101 Joensuu, Finland

e-mail: ilpo.laine@joensuu.fi 